\title{
Fly screen for flu targets
}

\section{By Michael J. Haas, Senior Writer}

A paper in Nature describes an RNAi screening strategy in Drosophila that could yield new host and viral targets to prevent or treat influenza infection, and possibly other viral infections. ${ }^{1}$ But opinions vary over whether the method is superior to use of human cell lines.

The research team included scientists from the University of Wisconsin-Madison, Howard Hughes Medical Institute, Airlangga University, the University of Tokyo and Kobe University. The team was led by Yoshihiro Kawaoka, professor of virology at Wisconsin-Madison and at Tokyo.

Kawaoka told SciBX that the screening method exploits two features of the Drosophila genome: it is smaller than mammalian genomes, allowing for more rapid screening; and it has a high degree of homology with mammalian genomes, including the human genome.

"Nearly all genes in the Drosophila genome

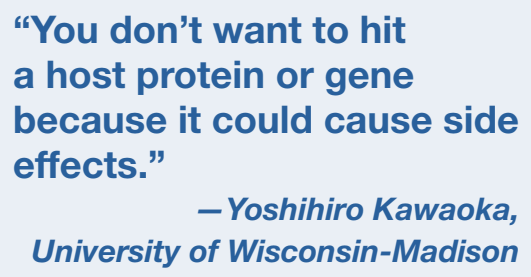

"You don't want to hit a host protein or gene because it could cause side - Yoshihiro Kawaoka, University of Wisconsin-Madison

of three known to localize to endosomes-membrane-bound compartments within cells through which influenza achieves entry. The team confirmed the virus's dependence on the three factors by silencing the genes with short interfering RNA (siRNA) in human embryonic kidney (HEK) cells. Silencing of the factors in cells infected with VSV or vaccinia virus had no effect, demonstrating that the screen had selectively identified host factors for influenza.

Collectively, the results showed that the RNAi screen of infected Drosophila cells could identify host factors that were potential new targets against influenza infection. However, Kawaoka said the team's ultimate purpose is not to identify new host factors as targets, but rather to use those host factors to identify new viral targets.

"You don't want to hit a host protein or gene because it could cause side effects," he said. "Next we need to find interactions between host and viral proteins that are essential to the virus. Then we could inhibit this interaction by targeting the viral proteins."

Kawaoka also said the screening method should be extendable to any human virus that infected, or could be modified to infect, Drosophila cells and that, like influenza, could complete at least part of its life cycle in Drosophila.

\section{But will it fly?}

Researchers contacted by SciBX disagreed over how relevant the screen was to influenza and other viral infections in humans, or whether the method had any advantages that would outweigh the limitations noted by Kawaoka.

have human homologs," he said. "Thus, something which is important to a virus in Drosophila is likely to be important in human cells, too."

\section{Flu fly factors}

Despite the potential advantages of using the Drosophila genome to identify new host factors, the team had to surmount a key obstacle: Drosophila cells lack the receptors the flu virus needs for infection.

Consequently, the team first had to develop a modified influenza virus capable of infecting Drosophila. To do so, they replaced hemagglutinin - a glycoprotein in the influenza virus envelope-with a glycoprotein from the envelope of vesicular stomatitis virus (VSV), which is capable of infecting Drosophila.

Next, Drosophila cells were infected with this modified virus and screened against a commercial RNAi library from Ambion, a subsidiary of Applied Biosystems. The library contains more than 13,000 doublestranded RNAs (dsRNA). Each dsRNA was complementary to, and therefore capable of silencing, a single Drosophila gene. The library covered about $90 \%$ of the Drosophila genome.

The screen identified 110 Drosophila host factors on which influenza depends for survival and replication. But, as the team reported in Nature, it found no evidence that the infected cells released virions to infect other cells, in part because Drosophila does not express all of the proteins required for virion assembly and infectivity.

Lastly, from these 110 factors the team selected the human homologs
Antonin de Fougerolles, senior director of research at Alnylam Pharmaceuticals Inc., said the work was "an important advancement in the understanding of the role of host factors in influenza infection."

"There is a lot of interest in the scientific community in using RNAi technology to help screen and identify viral host factors," he said, but in vivo studies of the identified host factors would be needed to validate them as potential therapeutic targets.

Alnylam and partner Novartis AG have ALN-FLU01, an RNAi-based vaccine, in preclinical development against pandemic influenza. Alnylam also has the specific siRNA ALN-RVS01 in a Phase II trial to treat respiratory syncytial virus infection.

By contrast, Amy Espeseth, director of RNAi therapeutics at Merck \& Co. Inc.'s Merck Research Laboratories unit, was skeptical that an RNAi screen in Drosophila would yield results that were meaningful to viral infections in humans.

"Many groups are doing genome-wide RNAi screens using human cells," she said. As an example, she cited a recent report in Science from Brigham and Women's Hospital and Harvard Medical School that identified over 200 host factors for HIV in an siRNA screen of human cells. ${ }^{2}$

"By using human cells, they were able to use a virus capable of completing a full cycle of replication and they could identify host factors that interact with the virus at all stages of its life cycle," she said. "I believe this offers an advantage over running siRNA screens in Drosophila cells." 
Andrew Henderson, associate professor of medicine at Boston University School of Medicine, said the Nature paper provided clear proof of concept for the method. But like Espeseth, he expressed doubt about whether it held any inherent advantages.

"In general, I like the concept, and the fact that this will identify general biochemical processes that viruses may require," he said. "The major concern that I see is that the screen is somewhat limited in what steps of the life cycle can be explored. It wasn't clear to me-especially since they are using cultured Drosophila cell lines-how much of an advantage this offers over an siRNA screen in avian or mammalian cells, which could support replication."

Henderson also was uncertain how extendable the method would be to other viruses, such as HIV.

"I think the cell- and species-specificity of HIV offers many unique challenges where using such an approach in flies may not be informative," he said. "This specificity has even created barriers in generating rodent models for HIV."

\section{No prevention}

In addition to the question of whether the method could identify new therapeutic targets against influenza, Gale Smith, VP of vaccine development at Novavax Inc., said that the method was unlikely to yield new targets for developing prophylactic vaccines against influenza.

"The very first thing a virologist or vaccinologist wants to know is how the virus interacts with the cell and enters it," he said. "The most important targets are those involved in those initial interactions."

Smith pointed out that Kawaoka's team replaced two glycoproteins in the influenza envelope that are of key importance to viral entry: hemagglutinin and neuraminidase, which was replaced with a fluorescent protein that provided a photometric readout during the RNAi screen.

"While the method is useful for looking at certain intracellular interactions between the virus and host," he said, "you miss the interactions of the cell with hemagglutinin and neuraminidase"-making it hard to see how the method could apply to preventive vaccine development.

"If this system could be used with intact viral envelope proteins, then you could possibly find host factors" with previously unknown roles in viral entry, Smith said. But he noted that mechanisms of viral entry have already been well studied in influenza and other viruses, such as HIV, "so it seems unlikely that something novel would come out of this approach."

Novavax has a virus-like particle (VLP) vaccine against the $\mathrm{H} 5 \mathrm{~N} 1$ strain of influenza in a Phase IIa trial that is expected to finish later this year. The company also plans to start a Phase IIa trial of a trivalent VLP vaccine against the $\mathrm{H} 3 \mathrm{~N} 1, \mathrm{H} 1 \mathrm{~N} 1$ and $\mathrm{B}$ strains of influenza this year.

\section{Targeting discussion}

Despite the lack of consensus on the utility and extendability of the screening method itself, companies did agree that identifying host factors was an important part of developing new therapeutics against influenza.

Alnylam's de Fougerolles said that targeting host factors was feasible, because such a strategy could be applicable to different subtypes of the virus. "With the advent of genome-wide RNAi-based screening, the number of potential targets will greatly expand," he said. "Establishing their validity as host targets for influenza infection, as well as their normal physiological roles, will be important in selecting which are appropriate therapeutic targets."

Merck's Espeseth agreed. "Although there are numerous strains of influenza, it is likely that most of the host proteins that are required for influenza infection are the same," she said. "Targeting the required host factors offers the advantage that the virus will be unlikely to evolve resistance to the drug."

She noted that while targeting host factors runs the risk of inducing toxic side effects, there are also risks in targeting viral proteins. "The strategy [proposed by Kawaoka] of targeting virus-host interactions is feasible as well, but doesn't provide the barrier to resistance that inhibiting a host factor does."

For now, Kawaoka said the team is studying the human homologs of the remaining host factors identified in their screen and determining the role each factor plays in supporting the life cycle of the virus. In particular, they are looking for interactions between host and viral proteins that are essential to the virus and thus offer new viral targets-but he said that the team had not yet identified any.

Kawaoka said that the University of Wisconsin-Madison has submitted a patent application for the findings reported in Nature.

\section{REFERENCES}

1. Hao, L. et al. Nature; published online July 9, 2008 ; doi:10.1038/nature07151

Contact: Yoshihiro Kawaoka, University of Wisconsin-Madison, Madison, Wisc.

e-mail: kawaokay@svm.vetmed.wisc.edu

2. Brass, A.L. et al. Science 319, 921-926 (2008)

\section{COMPANIES AND INSTITUTIONS MENTIONED}

Airlangga University, Surabaya, Indonesia

Alnylam Pharmaceuticals Inc. (NASDAQ:ALNY), Cambridge, Mass.

Applied Biosystems (NYSE:ABI), Foster City, Calif.

Boston University School of Medicine, Boston, Mass.

Brigham and Women's Hospital, Boston, Mass.

Harvard Medical School, Boston, Mass.

Howard Hughes Medical Institute, Chevy Chase, Md.

Kobe University, Kobe, Japan

Merck \& Co. Inc. (NYSE:MRK), Whitehouse Station, N.J.

Novartis AG (NYSE:NVS; SWX:NOVN), Basel, Switzerland

Novavax Inc. (NASDAQ:NVAX), Rockville, Md.

University of Tokyo, Tokyo, Japan

University of Wisconsin-Madison, Madison, Wis. 